\section{Adding Color To Grayscale Images}

Jerry Sedgewick

University of Minnesota

sedge001@umn.edu

In the world of fluorescent imaging, the need to colorize grayscale images can be an everyday task. Yet, when all too many of these colorized images go to publication, the results appear to contain undifferentiated blobs of a saturated color, rather than images that contain gradations of color.

While very small objects need to be as bright as possible in order to make these objects apparent (e.g. bacteria under low magnification), and while too many push the gain on photomultiplier tubes in order to top grayscale values off at 255 (on an 8-bit range) in order to show unquestionable colocalization, only the former can be defended on the basis of revealing lost features. The latter is done, it seems, because actual numeric measurements of colocalization often fall far short of what is both seen by eye and desired by the researcher, and it is far more beneficial to the researcher to show the saturated colors of red + green to yield large areas of yellow for the object of convincing both reviewers and readers (when most colocalization troubles concerning numeric measurements are best solved by reducing dark current noise with frame averaging versus yanking red and green values to saturated levels).

Add to this the cameras and confocal systems that collect grayscale images and then colorize these images red, green or blue, once more researchers are left with images that are colorized by unprintable colors. But these do look pretty on the computer screen.

Often these camera systems also show colorized images on a computer screen at video rates so that we can see the appropriate colors when acquiring fluorescent images. Yet, the eye does a much better job at seeing levels of gray (versus green or red or blue), and the eye can detect blobs of pure white much better than when images are colorized during the acquisition process. Thus, because we often acquire from live, colorized images, it is no wonder that so many images end up saturated: we can't make the right decisions when we can't see in the first place.

Acquisition of images. To the seasoned microscopist it goes without saying that white levels and black levels need to stay within the dynamic range of the acquisition system. Put more simply, the blacks can't be too black and the whites too white. Remember that blacks can always be made blacker and whites whiter in post-processing programs. Nothing can be retrieved from areas that are too white or too black, and these areas are difficult to work with when going to publication.

Often software acquisition programs contain a LUT (Look Up Table) overlay so that the user can visually discriminate areas that are saturated. These might show red, for example, where the whites are too white and green where blacks are too black. This overlay is not a part of the image, just something that covers the image to reveal saturation (based upon a mapping of saturated pixel values to a graphic overlay). The user is prompted, then, to adjust black level (or offset, or contrast) and white level (or gain, or brightness)

to rid the image of all

but 5 percent or so of these marker colors (assuming that these saturated pixels are a result of dark current noise and will be eliminated by using frame averaging).

The LUT overlay can be placed on grayscale images, or on colorized images when adjusting values during "live" acquisition. In that way, even though we cannot discriminate brightness well with colorized fluorescent images, we can overcome visual constraints with the LUT ov monitor-to-monitor differences, or the contrast and brightness settings on the monitor used, will not affect gain and black level settings versus settings determined by eye.

Once the LUT overlay is used to bring values within the dynamic range of your imaging system at the beginning of your microscopy session, then these values can often be retained for subsequent samples.

Adding Color. Even if you have acquired a colorized image, you may want to re-colorize it so that the colors fit the gamut of printers, and that of publishers. Be sure to use Channel Mixer under Image > Adjust(ments) to make the image monochrome (be sure to check the Monochrome box). The color green can still remain difficult to reproduce accurately for publishers, and I have been $90 \%$ successful. Occasional I get a green, "head-banger" image, and I can't put my finger on the source of this problem.

Once you have a grayscale image, then you are ready to add color: Under Image, select Mode to change the Mode from either Indexed Color or Grayscale to RGB Color. You may also have to change the bit depth from 16-Bit to 8-Bit.

2. Under Image on the menu, select Adjust(ments) then Curves. In the Curves dialogue box be sure that the gradient under the grid and line is black to the left and white to the right. If not, click the small arrowhead in the center of that gradient to swap the position.

3. Eliminate the colors you do not desire according to the following method In each instance, you drag the top of the line downward along the right edge until the output value reads as specified. Note that these output values are not set in stone, but the advantage of setting these in this manner lies in NOT completely eliminating unwanted colors: some of the other colors need to be included in order to get the best reproduction when printed.

To obtain Red colorized image:

Select Green Channel Set to Output
Select Blue Channel Set to Ouput of
obtain Green colorized image:

To obtain Green colorized image:

Select Red Channel

Set to Qutput of

Select Blue Channel Set to Output of

To obtain Blue colorized image:
Select Red Channel
Set to Output of
Select Green Channel
Set to Output of

You will note that the blue colorized image is closer to cyan. That is because many printers cannot print purple-blue images as well as green-blue images, and that is especially true when these images are published.

Save your finished image as a TIFF image for more universal use, and then, if you wish to drop the image into Powerpoint or Word, save the image as a JPEG (using Maximum for the JPEG setting).

A complete procedure for confocal imaging will be published as a book chapter in the 2nd edition of Confocal Microscopy: Methods \& Protocols by Author: Stephen W. Paddock edition 2 by Humana Press.
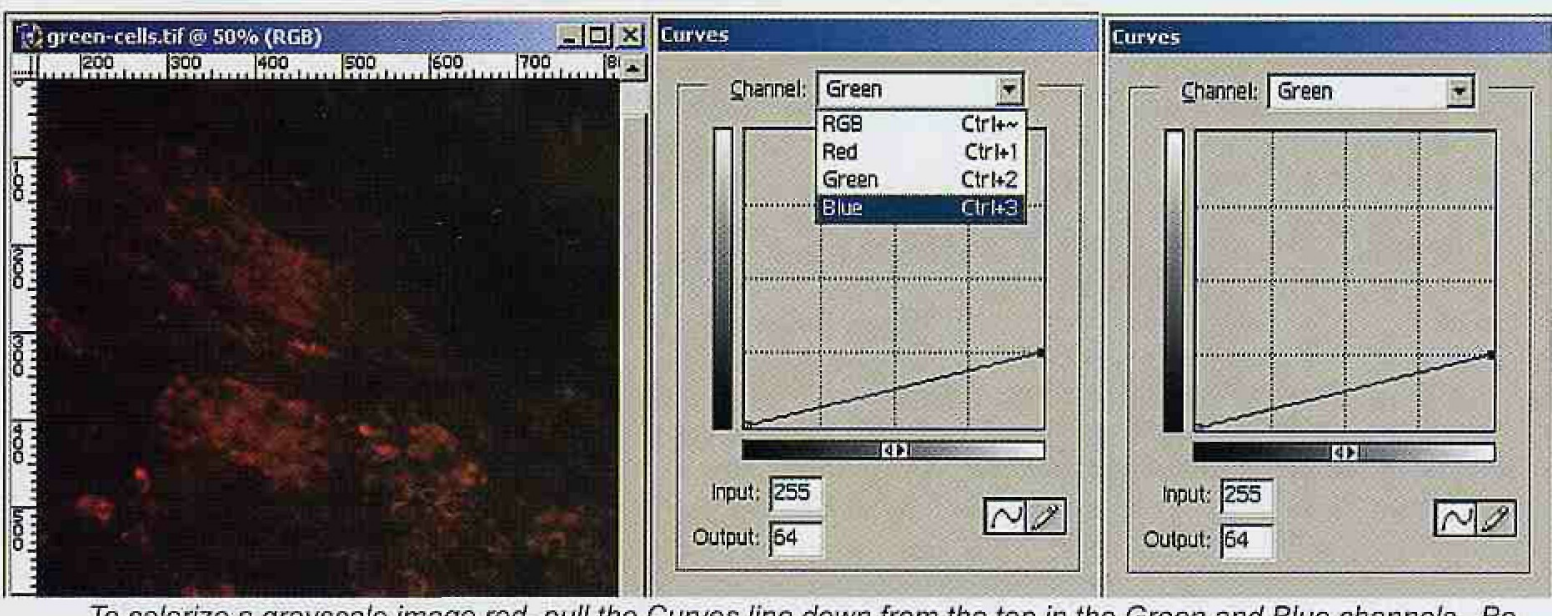

To colorize a grayscale image red, pull the Curves line down from the top in the Green and Blue channels. Be sure the grayscale gradient at the bottom of the Curves dialogue box shows black at the left hand side (accomplished by clicking on the small triangle at center). Pull down the Green to an Output value of -65 and the Blue to $\sim 40$. 